\title{
The design and validation of the R1 personal humanoid
}

\author{
Alberto Parmiggiani ${ }^{\alpha}$, Luca Fiorio ${ }^{\alpha}$, Alessandro $\mathrm{Scalzo}^{\alpha}$, Anand Vazhapilli Sureshbabu ${ }^{\alpha}$, \\ Marco Randazzo $^{\alpha}$, Marco Maggiali ${ }^{\alpha}$, Ugo Pattacini ${ }^{\alpha}$, Hagen Lehmann ${ }^{\alpha}$, Vadim Tikhanoff ${ }^{\alpha}$, \\ Daniele Domenichelli ${ }^{\alpha}$, Alberto Cardellino ${ }^{\alpha}$, Pierpaolo Congiu ${ }^{\beta}$, Andrea Pagnin ${ }^{\gamma}$, Roberto Cingolani $^{\delta}$ \\ Lorenzo Natale ${ }^{\alpha}$ and Giorgio Metta $^{\alpha}$
}

\begin{abstract}
In recent years the robotics field has witnessed an interesting new trend. Several companies started the production of service robots whose aim is to cooperate with humans. The robots developed so far are either rather expensive or unsuitable for manipulation tasks. This article presents the result of a project which wishes to demonstrate the feasibility of an affordable humanoid robot. $R 1$ is able to navigate, and interact with the environment (grasping and carrying objects, operating switches, opening doors etc). The robot is also equipped with a speaker, microphones and it mounts a display in the head to support interaction using natural channels like speech or (simulated) eye movements. The final cost of the robot is expected to range around that of a family car, possibly, when produced in large quantities, even significantly lower. This goal was tackled along three synergistic directions: use of polymeric materials, light-weight design and implementation of novel actuation solutions. These lines, as well as the robot with its main features, are described hereafter.
\end{abstract}

\section{INTRODUCTION}

The recent years have seen a great increase in robotics applications, with several companies startng to deliver marketready systems. The development of robots intended to interact with humans is becoming a very important sector, with the main obstacles to the market remaining: cost and technology complexity. Several robot manufacturers and suppliers have started to address these issues concentrating their efforts in the development and supply of low-cost platforms ${ }^{1}$. These can be subdivided in two main categories: i) mobile base manipulators and ii) fixed base robots.

The best known examples of the former category are Fetch, Pepper, Tiago and HSR. Interestingly, besides Pepper, most mobile manipulators are single-arm systems; this design choice is most probably dictated by the requirement to limit the overall systems' cost and complexity.

Fetch Robotics announced in April 2015 its Fetch and Freight robots [1] whose intended initial applications were

${ }$ iCub Facility, Fondazione Istituto Italiano di Tecnologia, Via Morego 30, 16163, Genoa, Italy; alberto.parmiggiani@iit.it, giorgio.mettadit.it

$\beta$ drop, Calle Castella 33 esc. B2 2-3, 08018 Barcelona, Spain; p.congiuddrop-innovation. com

$\gamma_{6.14}$ creative licensing, via P. Lomazzo 19 c/o JWT, 20154 Milano, Italy; apagnin@ 6punto14. com

$\delta$ Fondazione Istituto Italiano di Tecnologia, Via Morego 30, 16163, Genoa, Italy; roberto.cingolanieit.it

${ }^{1}$ Given the lack of scientific papers and published technical reports we resorted to citing product websites for the current section. All robot costs are reported to the best of our knowledge and are to be intended as an approximate reference. All cited websites were accessed on February $28^{\text {th }}$ 2017.

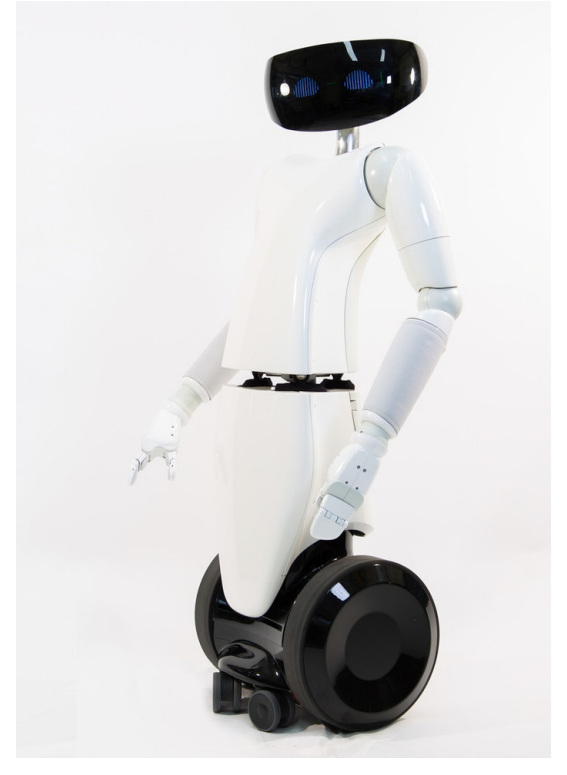

Fig. 1. The R1 humanoid robot.

automated logistics, material handling and warehouse management; recently Fetch Robotics started offering the Fetch robot as research platform. The sales price of the Fetch mobile manipulator has not been disclosed but it has been reported to be “less than $\$ 100,000$ " [2].

Softbank robotics released its Pepper [3] robot in June 2014. The robot is being made available through a lease plan at approximately $9.300 €$ for 36 months [4]. Pepper is mostly intended as a communication device, and as its developers state [5]: "The Robot cannot carry objects - we designed Pepper to gesticulate, hug and express himself only".

Tiago [6] (developed by PAL robotics) is a one-armed low-cost robot based on the REEM technology that was announced in February 2015. Tiago is mainly intended for research and academic applications; starting from the robot datasheet we estimate the cost of the robot should be in the $30.000 €-60.000 €$ range.

Recently Toyota unveiled the second version of its human assistance robot (HSR) [7]. The robot was first unveiled in September 2012, and its newer version with a simplified 4DOF arm was announced in July 2015. A sale price of the HSR has not been announced although sources from Toyota recently stated it will be made available to research institutions for rent at a cost of $950 \$ /$ month. 
A final robot to be cited is Care-O-bot 4 [8] announced in January 2015 by Fraunhofer IPA. Like Tiago, Care-O-bot 4 is mainly intended for research. We estimate the cost of the two-armed robot version should be in the order of $200.000 €$, considering its arms are based on the 7DOF LWA 4P Schunk arms and that it comprises a sophisticated sensor array.

While the robots mentioned above are all mobile manipulator systems some companies have started offering affordable fixed base robots.

Among these we can cite Baxter [9] released in 2012 by Rethink Robotics and YuMi [10] by ABB announced in 2011, which were developed mainly for assembly and light manufacturing tasks. Danish constructor Universal Robots also focuses on the same market segment with its line of costeffective manipulator arms (UR3, UR5 and UR10), released in 2009 .

Starting from these premises, in January 2015 we decided to undertake a new project to make the iCub technology [11] accessible to everybody. This eventually led us to develop the $\mathrm{R} 1$ robot whose characteristics are described hereafter.

This paper is structured as follows. Section II presents the design requirements and Section III the most important design choices we made to implement the former. The robot hardware is described in detail in Section IV; Section V presents a validation of our design approach and is followed by the conclusions.

\section{REQUIREMENTS}

The high level goal of the project was to demonstrate the feasibility of an affordable humanoid robot, with good manipulation capabilities.

We approached the design of R1 by employing well established product design practices [12]. The design started from a general list of high-level user needs. We wanted the robot to be very affordable, to allow for easy and natural interaction, to have an elegant and glossy look, to grasp, move and manipulate objects, to operate switches and to open doors, and to be capable of safely navigating its environment. This list was then transformed into detailed specifications to be implemented in the successive design phases.

We believe the first applications of service robots for assistance applications are likely in "structured" environments such as offices, airports, hotels, retail spaces etc. Most probably service robot providers will adopt a business-tobusiness (B2B) business model. This lead us to hypothesize that $12000 €-16000 €$ would be an appropriate cost range for the production of a large batch of robots (e.g. 2000 units), leaving sufficient margin for a robot supplier to operate with an appropriate profit/cost ratio.

\section{DESIGN RATIONALE}

Differently from how we approached the design problem for the $\mathrm{iCub}$ [11], we began the design of R1 starting from its desired target cost. Production costs of most goods are heavily affected by the size of the production batch size: the more are made the lower their unit cost. A first challenge were the conflicting requirements of designing and constructing a robot with technologies suitable for prototypes while demonstrating its feasibility and cost effectiveness in a large scale production scenario. A second challenge we faced was how to negotiate cost vs. functionality trade-offs. Assistive service robots are not currently available nor do we know with precision which tasks they will be required to preform. Therefore it was difficult to decide when the additional costs for high-performance components and parts were justified. Throughout the duration of the project we tried to resolve these "conflicts" by favouring the limitation of the overall system's costs, and to resist the natural tendency to overengineer in situations of uncertainty. At the early stages of the design process we took a series of important design decisions, that are described in the following subsections.

\section{A. Components selection}

We carefully selected commercial off-the-shelf (COTS) component to be integrated with in-depth evaluations of cost/benefits trade-offs To support the process of COTS component selection we collected data regarding the cost (and possible discount rates vs. quantity) for components commonly employed in robots (e.g. bearings, transmissions, embedded CPU, encoders, motors, RGBD scanners, etc.). Knowing the approximate number of DOF, and the type and number of components we were likely to employ for every robot part, we identified possible component combinations that allowed us to obtain the desired functionalities while limiting the overall system cost.

\section{B. Polymeric materials}

In designing the most important sub-assemblies we deliberately chose to employ engineering plastics as construction materials for most frame components and some mechanical transmissions. This choice was dictated by the need to demonstrate the feasibility of an affordable platform. The materials we chose to employ are plastics that can be processed by injection moulding, which is among the most cost-effective production processes when lot sizes exceed the thousands threshold. The consequences of this choice are discussed in greater detail in Section V.

\section{Wheeled locomotion}

We decided to endow R1 with wheels instead of legs to enable locomotion. The use of wheels allows to reduce the number of DOF, hence the complexity and cost of the platform. Moreover wheeled robotic platforms can be statically stable, and characterized by simpler, more predictable dynamics. Using wheels instead of legs poses issues for the traversal of stairs, gaps, and small ridges. The robot should nevertheless be capable of navigating structured indoor environments (e.g. museums, hospitals, schools, shopping malls). We speculate these capabilities will probably be sufficient for developing applications that deliver value to end-users. 


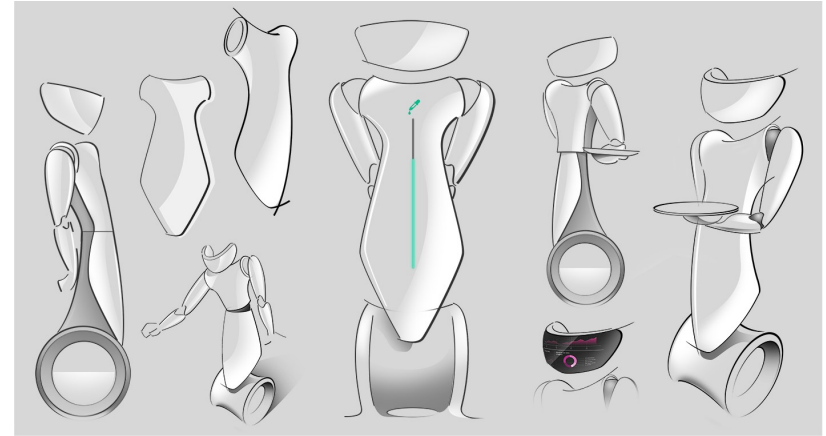

Fig. 2. Style concepts. The figure represents the initial concept sketches that were used to develop the robot aesthetics.
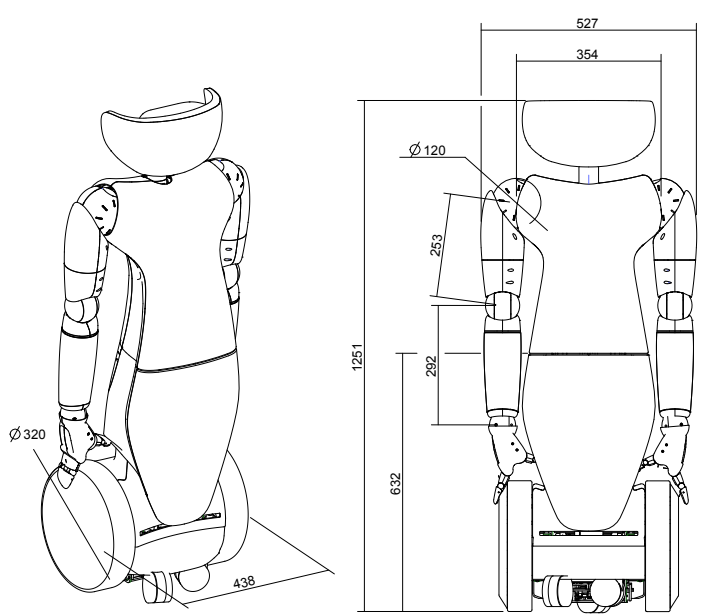

Fig. 3. Robot dimensions. The figure shows the main dimensions of the robot in its home configuration. All dimensions are in $\mathrm{mm}$.

\section{Styling}

To obtain a pleasing aesthetic effect we worked with product designers and styling professionals. The robot was conceived with the aim of generating empathy with the user through a friendly and elegant design with pure and sinuous surfaces. The image of the robot is characterized by combining elements and solutions inspired by the human body, such as arms, body postures and gestures, with other original elements that respond efficiently and elegantly to functional requirements. We initially defined a tentative volume allocation for most components and used that as a basis to develop the initial style of the robot (some of the original concept sketches are represented in Fig.2). We then proceeded with the detail design phases and with frequently iterations we adapted the style of the robot. Finally the design of the robot was protected internationally via a registered Community design [13] obtained through the European Union Intellectual Property Office (EUIPO).

\section{ROBOT HARDWARE}

\section{A. Overview}

The main robot dimensions are shown in Fig.3; its joint structure is shown in Fig.11 and joint parameters are listed in Table II. The robot is $1.2 \mathrm{~m}$ tall. The robot is designed with an

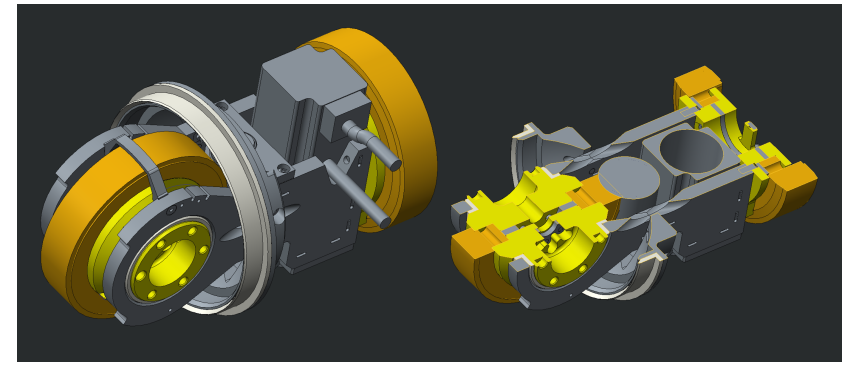

Fig. 4. Shoulder pitch-roll assembly. The figure shows a CAD view of the right shoulder assembly and its cross section. Plastic bushings (represented in white), high resistance plastic plates and shafts (represented in yellow), and igus RL-D-50 gears (represented in orange) are clearly visible in the picture.

especially small footprint to move in cluttered household and office environments. The footprint of the robot is a rectangle $400 \mathrm{~mm}$ wide and $350 \mathrm{~mm}$ long. The support area of the robot is increased with three pivoting castor wheels. The robot is designed to be lightweight, $54 \mathrm{~kg}$ in total (including batteries). This allows for easier transportation and makes the robot safe to interact with.

The robot is equipped with active force-torque control and exploits measurements from two six-axis force torque sensors in its arms, and tactile sensors in its hands and forearms to generate safe and compliant motions. All covers are completely closed with no pinch points. These features are intended safe human-robot interaction as dictated by the ISO13482 standard [14].

The robot moves by means of two driving wheels. Its maximum speed is limited to $0.6 \mathrm{~m} / \mathrm{s}$ in software not to pose risks to users' safety. The robot has two 8DOF arms., that are capable to protrude and elongate when needed. The payload of the robot arm is $1.4 \mathrm{~kg}$ with arms stretched (but without arm elongation), reaching at a $0.6 \mathrm{~m}$ distance from the robot body. Higher payloads are obtainable at shorter distances. Upper arm joints are driven by actuators comprising Mecapion APM-SA0 100W BLDC motors, and polymeric worm gears by igus (models RL-D-50 and RL-D30 ). A CAD view of the right shoulder assembly is show in Fig.4.

The robot torso is equipped with a torso mechanism that allows it to vary its height from a minimum of $1.15 \mathrm{~m}$ to a maximum of $1.35 \mathrm{~m}$. This allows it to adapt its height to the height of the surface on which it is manipulating (see Sec.IVC). The same mechanism was integrated in the forearm of the robot to allow the robot to extend its arms and pick up objects from the floor or high shelves. Further details regarding the design of the end-effectors are available in [15].

\section{B. End-effectors}

The robot has two four-DOF, two degrees of actuation (DOA) hands. The robot hands were designed to allow opening of different types of doors (doors with handles, doors with push bars, etc.) Each hand has four phalanges, coupled two-by-two and driven by low friction Spectra tendons each pulled by a DC geared motor (Canon DG16L), which can 


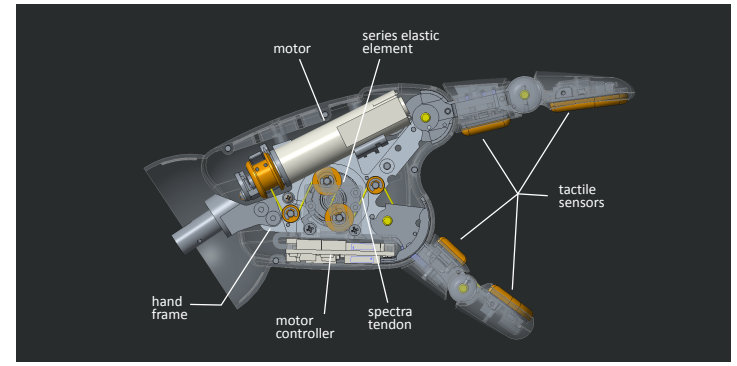

Fig. 5. Hand. The figure shows the R1 robot hand and the main components it comprises.

generate approximately $20 \mathrm{~N}$ of normal force at the outermost part of the distal phalanges. Most of the parts of the hand are made of polymeric materials with the exception of the springs and the joints shafts. Differently from the robot hands we designed so far, plastic bushings were used in each joint instead of precision ball bearings. The return of the phalanges to the open position is achieved with torsional springs located at the hand joints. The hands are equipped with distributed pressure sensors, joint angle encoders and series elastic actuators to allow monitoring gripping forces. The pressure sensors are based on the iCub skin technology [16]. The gripping force is measured with two series elastic element (SEE) inspired on the work by Ito et al. [17]. The actuation tendons wind through the SEEs that are placed between the motors and the joints. This enables the hand to sense forces and simultaneously offers protection from impact forces. The hand hardware is completely self-contained, comprising all needed control boards and motors; it is therefore easy to attach and interface to the robot's wrist. In the future this may allow utilizing different sets of end effectors depending on the application area.

\section{Parallel mechanisms}

The robot features three parallel mechanisms: one in the lower torso to move the chest and one in each forearm to actuate the wrist. Each parallel mechanism has three DOF, namely, extension, roll and pitch. The design of the mechanism is adapted from a previous work proposed by Bussola et al. [18], and includes a base, three linear actuators and a platform. Each actuator is fixed perpendicularly to the base on the vertices of an equilateral triangle, and is connected to the platform through a passive spherical joint followed by a passive prismatic joint. The major advantages of this solution are:

- high payload and high structural stiffness compared to serial chains

- light platform thanks to the arrangement of the actuators at the base

- simple kinematic structure, that allows computing both the forward and the inverse kinematics in terms of closed-form expressions

The torso mechanism has been designed using MOTECK LD20 COTS linear actuators. The actuators comprise a DC motor that drives a lead screw connected to a rod, and can

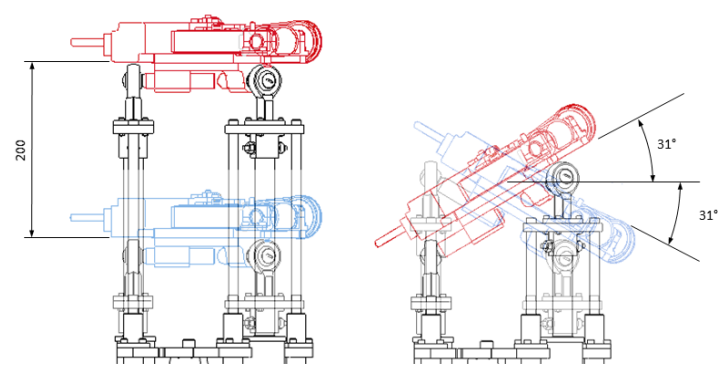

Fig. 6. Tripod mechanism. The figure shows the tripod mechanism of the torso in two different configurations that represent its range of motion.

provide a peak force of 400 [N]. Furthermore, as depicted in Fig.6, a custom frame provides the structural stiffness, while each actuator has been endowed with an adjacent linear guides to protect the rod from radial loads.

The wrist mechanism employs custom designed linear actuators. Each actuator consists of a DC geared motor from CANON and a lead screw with plastic nut. Similarly to the torso, the position of the nut is computed by means of a rotary encoder mounted on the screw; custom linear guides have been designed to bear radial forces.

For more details about the range of motion and peak effort of each joint refer to Table II in the Appendix; further details regarding these mechanisms are available in [19].

\section{Manipulation workspace}

The shape and size of the robot were determined starting from the manipulation requirements described in the Sec.II. We were particularly interested in allowing the robot to manipulate with ease on horizontal planes of different heights (tables heights normally found in households and offices typically vary in the $0.7 \mathrm{~m}$ to $0.9 \mathrm{~m}$ range).

The workspace of the robot is limited by two constraining factors: i) the limitations of joint ranges of motion (ROM) and ii) static stability. The static stability constraint prevents the robot to reach for configurations where its arm is distant from its body: when the projection of the centre of gravity (COG) moves close to the limit of the support polygon, the robot risks to topple and fall.

To verify which points can be reached safely we conducted a reachability analysis, where we explored reachable points on two horizontal planes placed at $0.7 \mathrm{~m}$ and $0.9 \mathrm{~m}$ from the floor reference plane.

On these two planes we selected a set of target points, defined with respect to a polar coordinate system centered in the principal vertical axis of the robot. Points were spaced $25 \mathrm{~mm}$ apart in the radial direction and $2.5^{\circ}$ apart in the angular one. The hand orientation was constrained so that the hand remained vertical, i.e. the normal to the palm is orthogonal to the normal of the horizontal planes This orientation was selected as the most representative for manipulation objects lying vertically on tables (e.g. cups, bottles, books). We locked the torso yaw joint so as to keep the torso oriented in the forward direction. 

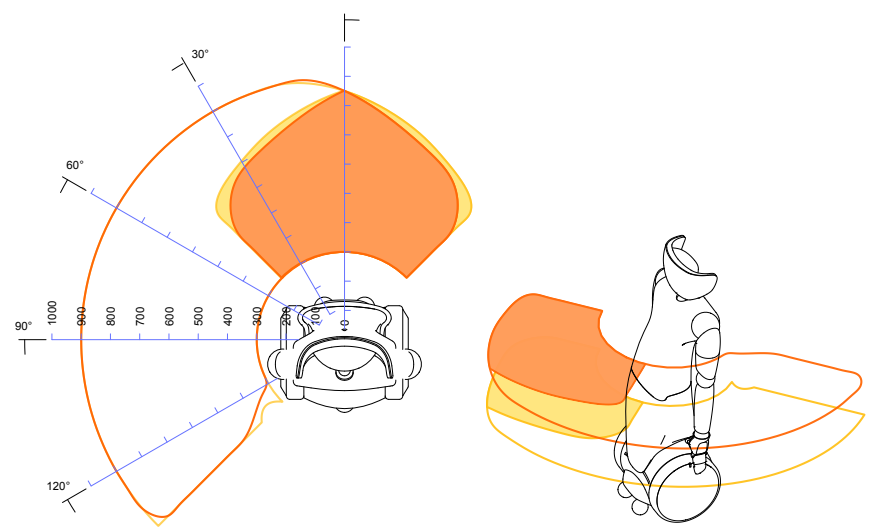

Fig. 7. Reachable workspace. The figure shows the area that is safely reachable by the left end-effector, in two horizontal planes placed at $0.7 \mathrm{~m}$ and $0.9 \mathrm{~m}$. The area overlapping with the reachable area of the right endeffector (where bimanual manipulation can take place) is represented as shaded. Distance ticks are labeled in $\mathrm{mm}$; as can be seen the robot can reach at $0.9 \mathrm{~m}$ in almost all angular positions. Notice that the reachable regions on the two planes are rather similar: this is an effect of the torso tripod mechanism that allows the robot to vary its height.

The inverse kinematics solver was then requested to reach all the points while keeping the COG of the robot $40 \mathrm{~mm}$ away from the limit of the support polygon. The test was conducted considering a $2 \mathrm{~kg}$ load applied at the end effector.

Additionally we wanted to determine where the arms could reach and how wide was the region where the robot was capable of manipulating bi-manually. Fig.7 represents the results of these simulations; coloured envelopes define the regions that can be reached successfully.

\section{E. Head}

The robot head and its "face" are possibly the most important interface in human-robot interaction (HRI). The head integrates a custom designed curved, programmable RGB LED display that allows to facial expressions. Since we wanted to render images on a double curvature surface we resorted to a design largely inspired on the work by Willis and colleagues [20]. The display comprises five custom designed $32 \times 16$ RGB LED matrices, driven by a Xilinx Zynq®, which were joined and used to backlit a 3D printed setup. This setup consisted of arrays of optical relay tubes with a count of one light pipe for every individual LED in the display matrix (2560RGB pixel in the current design). To better separate the light emitted by the "pixels" and to guide the light pipe, another mechanical element made in black polyethylene was employed. The entire construction was then covered by a methacrylate mask which increases true blacks and accentuates the transmitted light, while acting as the projection curved surface. Further details can be found in the dedicated article by Lehmann et al. [21].

\section{F. Electronics}

The robot embeds battery and BMS, computing units, motor control boards and different kinds of sensors. The robot battery is a Lithium based $25.4 \mathrm{~V} 15 \mathrm{Ah}$ pack with a battery managment system (BMS) installed on its base.

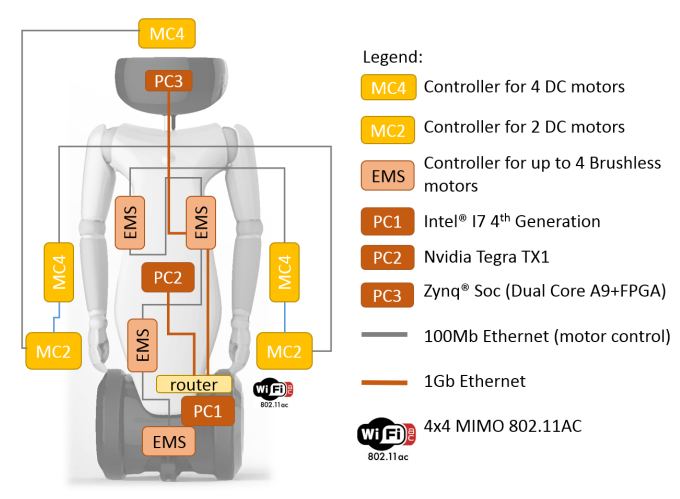

Fig. 8. Robot electronics layout. The diagram represents the placement of the main electronic components and the topology of the connecting buses.

A custom board provides and controls the main battery output $(25.4 \mathrm{~V}$ nominal) and a $12 \mathrm{~V}$ regulated output. $\mathrm{R} 1 \mathrm{has}$ one Nano-ITX Intel 77 computing unit (PC1), installed in the base, one Nvidia Tegra TX1 in the torso (PC2), and a Xilinx ZynQ based board (PC3). All these boards are connected via a $1 \mathrm{~Gb} / \mathrm{s}$ LAN to the EA-AC87 ASUS $5 \mathrm{GHz}$ Wireless-AC 1800 access point (see Fig.8). PC1 is the master of the $100 \mathrm{Mb} / \mathrm{s}$ Ethernet bus that connects all the motor control cards, reads a Robopeak RPLidar laser sensor and the distance sensors on the base. The PC2 controls the video system, based on the Leopard Imaging OV580 stereo camera module and a an Xtion Pro Live RGBD sensor PC3 has been customly designed to control the RGB Led Matrix and the loudspeakers audio amplifier, but also to acquire data from a 9 axis IMU. Moreover it can acquire up to 8 MEMS microphones for beamforming purposes.

Most motor control boards sport embedded accelerometers and gyroscopes which allow to monitor the state of the robot and to quickly identify the occurrence of unplanned impacts. The hands and forearms of the robot are equipped with the pressure-sensitive skin technology developed for the iCub project [16]. The robot has two six-axis force-torque sensors installed in its arms to measure and control physical interaction.

\section{VALIDATION}

Despite excellent techno-polymers have been synthesized in recent years, their mechanical properties are still far from those of most construction metals (see Table I for an overview). On the other hand, the density of engineering plastics is significantly lower than that of metals. Indeed, high grade plastics and standard aluminum are rather close in terms of strength-to-weight ratio. Hence engineering plastics components can provide resistance levels close to those of aluminum provided that enough material is employed. As a consequence, the weight of the robot cannot be significantly reduced, as addition of material is required to compensate the loss in strength.

Similar considerations hold for the stiffness of components. Metals are characterized by moduli of elasticity up to two orders of magnitude greater than those of plastics. 
TABLE I

MATERIAL DATA. THE TABLE SHOWS A COMPARISON OF THE MECHANICAL PROPERTIES OF STANDARD CONSTRUCTION METALS, AND ENGINEERING Plastics. Data Were extracted from the Matweb on-Line Data base.

\begin{tabular}{llcccc} 
material type & material designation & $\begin{array}{c}\text { Tensile Strength } \\
{[\mathrm{MPa}]}\end{array}$ & $\begin{array}{c}\text { Modulus of Elasticity } \\
{[\mathrm{GPa}]}\end{array}$ & $\begin{array}{c}\text { density } \\
{\left[1 \mathrm{e}^{3} \mathrm{~kg} / \mathrm{m}\right]}\end{array}$ & $\begin{array}{c}\text { Specific strength } \\
{[\mathrm{kNm} / \mathrm{kg}]}\end{array}$ \\
\hline High grade steel & 17-4PH / UNS J92150 & 1378 & 204 & 7.8 & 176.7 \\
Standard steel & C40 / AISI 1040 & 370 & 200 & 7.84 & 47.2 \\
High grade aluminum & A17075 & 462 & 71.7 & 2.81 & 164.4 \\
Standard aluminum & A16082 & 250 & 70 & 2.7 & 92.6 \\
Fiber reinforced Nylon & PA6.6 + GF30 & 160 & 11 & 1.35 & 118.5 \\
Nylon & PA12 & 66 & 2.4 & 1.03 & 64.1 \\
Acetal / Delrin & POM-C & 65 & 3.0 & 1.41 & 46.1 \\
\hline
\end{tabular}

This lack of stiffness can be compensated by appropriately increasing resistant sections. Finally whenever additional strength was required we combined engineering plastics with sheet-metal components.

Nevertheless these considerations prompt for a thorough verification of the robot's structural behaviour. We characterized these aspects with two specific tests: i) a robot configuration error test and ii) a repeatability test. In both cases the ground-truth configuration of the robot was measured with a Vicon ${ }^{\circledR}$ motion capture system, with sub-millimetre accuracy.

In the robot configuration error test we measured the end-effector pose errors caused by elastic deformations. The robot configuration measured with the robot's joints encoders was compared to the one measured with via motion capture. This comparison was performed in five different configurations; for each configuration ten repetitions were performed. Fig.9 shows the configuration with the largest error and its magnitude relative to the robot: as can be seen errors are significant.

In the repeatability test we measured to what extent endeffector pose repeatability was affected by elastic deformations. We considered five different configurations and acquired data in ten repetitions. Repeatability was rather good (See Fig.10), with errors under $2 \mathrm{~mm}$ for all tested configurations.

These experiments yield the preliminary indication that the end-effector configuration measured by the robot is not alone sufficient for reaching with the precision needed for manipulation tasks. Hence more advanced planning and control strategies, taking in account the cameras and RGBD feedback, will be needed to control the arms' movements.

\section{CONCLUSions}

In this paper, we presented the design and validation of a new affordable humanoid robot. The cost of the prototype was of approximately $70.000 €$. The cost of commercial components, around $12.500 €$, accounts for approximately $18 \%$ of the total cost. Assembly man-hour costs account for approximately $10 \%$ of the total costs. Constructing the custom mechanical parts for the prototype required approximately $50.000 €$.

Our estimates indicate that by exploiting the economics of mass production we could bring the cost of commercial components down to approximately 5.000€ and the cost of custom mechanical parts down to $8.000 €-10.000 €$ (with savings in the order of $80 \%$ thanks to improved manufacturing processes).

If the design is optimized for ease of assembly we expect the total cost of the robot could be reduced to the $15.000 €$ $-18.000 €$ range.

The use of materials and components for suitable for large scale manufacturing has proven to be a viable alternative, provided corrective measures are taken in controlling the robot's movements.

The robot prototype allowed us to perform simple reaching tests that confirmed the validity of our approach. The next phase of the project will be focused on the improvement of the robustness and dependability of the robot.

\section{ACKNOWLEDGMENTS}

The authors would like to thank:

- Luigi Focanti and the people at 6.14 and drop for the effort on the development of the style and expressiveness of the robot;

- Roberto Puddu, Marco Pinaffo, Charlie Sangiuneti, Massimiliano Mosto, Marco Giordano, Angela Morelli for the construction and assembly of mechanical components and Lorenzo Orciari for the support on the mechanical design;

- the people at Parametric Design, Telerobot Labs, and Mechadept for the help on the design of the robot;

- Francesco Rea and Alessandra Sciutti for their contributions and suggestions on the robot HRI features;

- Fabrizio Larosa, Davide Gandini, Simone Michelini and Emiliano Barbieri, for the logistic support and the help on wiring the system;

- Maurizia Botti for the support in developing the robot textile covers;

- Marco Accame, Valentina Gaggero, and Andrea Ruzzenenti for the development of the robot software;

- Filippo Biggi for the painstaking help on the construction and assembly of the robot.

Finally the authors would like to thank the anonymous reviewers that helped to improve the paper. 


\section{REFERENCES}

[1] Fetch Robotics, "Fetch research."

[2] IEEE Spectrum, "Fetch Robotics Introduces Fetch and Freight: Your Warehouse Is Now Automated."

[3] Softbank Robotics, "How pepper works."

[4] Softbank Robotics, "Launch sales of Pepper.."

[5] Softbank Robotics, "Top 100 questions."

[6] PAL Robotics, "Tiago."

[7] I. Toru, "Traveling robot, motion planning method for traveling robot, and storage medium storing program for traveling robot," 2014.

[8] Fraunhofer IPA, "Care-O-bot 4 website."

[9] Rethink Robotics, "Baxter robot website."

[10] ABB, "YuMi robot website."

[11] A. Parmiggiani, M. Maggiali, L. Natale, F. Nori, A. Schmitz, N. Tsagarakis, J. S. Viktor, F. Becchi, G. Sandini, and G. Metta, "The design of the iCub humanoid robot," International Journal of Humanoid Robotics, vol. 9, 2012.

[12] K. T. Ulrich, S. D. Eppinger, and A. Goyal, Product design and development, vol. 2. McGraw-Hill New York, 2011.

[13] A. Parmiggiani and G. Metta, "European Design EP RCD 003074913," 2016.

[14] ISO 13482, "Robots and robotic devices - Safety requirements for personal care robots," 2014.

[15] A. V. Sureshbabu, M. Maggiali, G. Metta, and A. Parmiggiani, "A parallel kinematic wrist for the R1 humanoid robot," in Proc. of Int. Conf. on Advanced Intelligent Mechatronics (AIM), 2017.

[16] P. Maiolino, M. Maggiali, G. Cannata, G. Metta, and L. Natale, "A flexible and robust large scale capacitive tactile system for robots," Sensors Journal, IEEE, vol. 13, no. 10, pp. 3910-3917, 2013.

[17] N. Ito, J. Urata, Y. Nakanishi, K. Okada, and M. Inaba, "Development of very small high output motor driver for realizing forceful musculoskeletal humanoids," in IEEE/RAS Int. Conf. on Humanoid Robots (HUMANOIDS), pp. 385-390, Dec 2010.

[18] R. Bussola, R. Faglia, and G. Incerti, "A tripod parallel robot as an active suspension for low frequencies damping," in Proc. 11th Int. Workshop on Robotics in Alpe-Adria-Danube region, 2002.

[19] L. Fiorio, A. Scalzo, L. Natale, G. Metta, and A. Parmiggiani, "A parallel kinematic mechanism for the torso of a humanoid robot: design, construction and validation," in Proc. IEEE/RSJ Int. Conf. on Intelligent Robots and Systems (IROS), 2017.

[20] K. Willis, E. Brockmeyer, S. Hudson, and I. Poupyrev, "Printed optics: $3 \mathrm{~d}$ printing of embedded optical elements for interactive devices," in Proceedings of the 25th annual ACM symposium on User interface software and technology, pp. 589-598, ACM, 2012.

[21] H. Lehmann, A. V. Sureshbabu, A. Parmiggiani, and G. Metta, "Head and face design for a new humanoid service robot," in International Conference on Social Robotics, pp. 382-391, Springer, 2016.
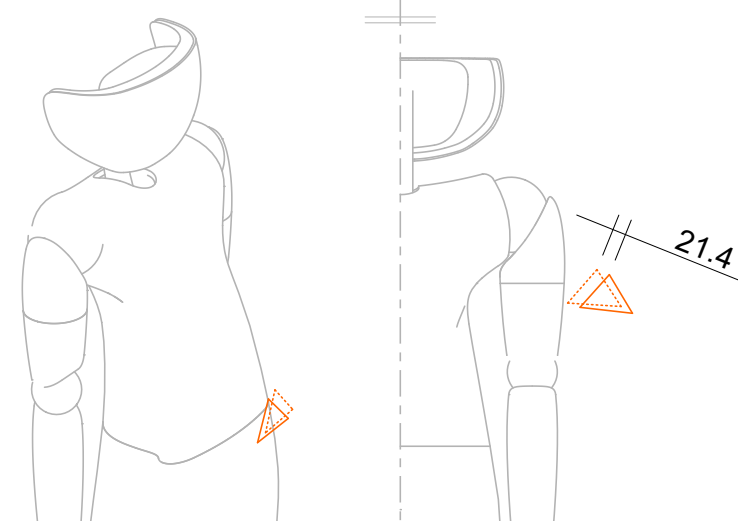

Fig. 9. Configuration error. The figure shows a three-dimensional plot of the end-effector configuration error in a typical manipulation configuration. The data presented are an average of 15 reaching movements from the arm's rest position. The actual robot end-effector configuration is represented by the solid triangles; dashed triangles represent the robot end-effector configuration measured by joint encoders. The average configuration error is rather large, well above $20 \mathrm{~mm}$.

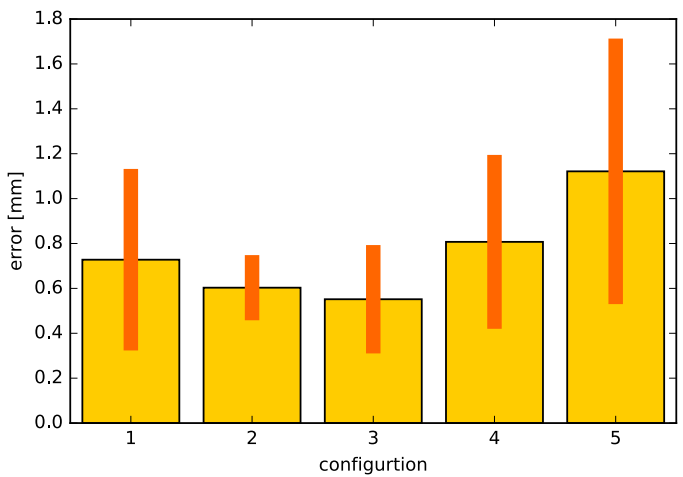

Fig. 10. Repeatability test. The figure shows a histogram plot of endeffector reaching errors for five representative arm configurations. Orange bars represent standard deviations. The test was conducted with 15 repetitions of the same arm movements. As can bee seen from the plots the same positions are reached with maximum deviations in the order of $2 \mathrm{~mm}$.

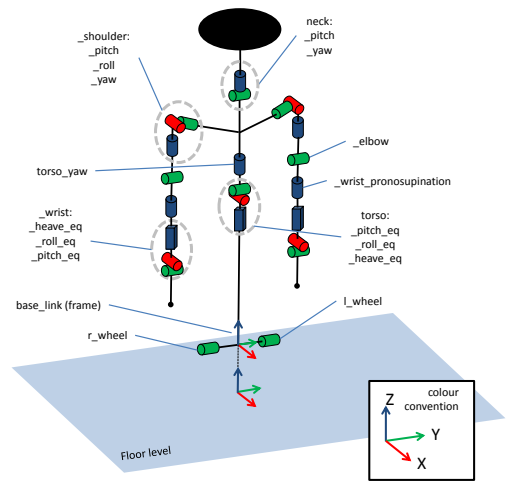

Fig. 11. Robot kinematics. The figure shows a representation of the robot kinematics. Tripod joints are represented with a "heave-pitch-roll" equivalent representation.

TABLE II

JOINT PARAMETERS. THE TABLE LISTS THE RANGE OF MOTION (ROM) AND PEAK EFFORT OF THE ROBOT JOINTS. THE ROM OF SERIAL JOINTS IS DEFINED BY HARDWARE LIMITS. THE ROM OF THE EQUIVALENT JOINTS “_EQ” IS ENFORCED AT THE FIRMWARE LEVEL.

\begin{tabular}{|c|c|c|c|c|}
\hline \multirow[t]{2}{*}{ CHAIN } & \multicolumn{2}{|l|}{ JOINT } & \multicolumn{2}{|c|}{ PARAMETERS } \\
\hline & Name & Type & $\begin{array}{c}\text { RoM } \\
\text { [deg] or }[\mathrm{m}]\end{array}$ & $\begin{array}{l}\text { Peak Effort } \\
{[\mathrm{Nm}] \text { or }[\mathrm{N}]}\end{array}$ \\
\hline \multirow{6}{*}{ BASE } & left_wheel & ROT & - & 16 \\
\hline & right_wheel & ROT & - & 16 \\
\hline & torso_heave_eq & PRIS & $0.0 /+0.17$ & 1200 \\
\hline & torso_roll_eq & ROT & \pm 30 & 70 \\
\hline & torso_pitch_eq & ROT & \pm 30 & 70 \\
\hline & torso_yaw & ROT & \pm 60 & 26 \\
\hline \multirow{4}{*}{ HEAD } & neck_pitch & ROT & $+50 /-30$ & 1.4 \\
\hline & neck_yaw & ROT & \pm 80 & 1 \\
\hline & shoulder_pitch & ROT & $-30 /+90$ & 26 \\
\hline & shoulder_roll & ROT & $-10 /+75$ & 26 \\
\hline \multirow{6}{*}{ ARM } & shoulder_yaw & ROT & \pm 90 & 7.5 \\
\hline & elbow & ROT & $0 /+100$ & 17.4 \\
\hline & wrist_pronosupination & ROT & \pm 90 & 2 \\
\hline & wrist_heave_eq & PRIS & $0 /+0.13$ & 126 \\
\hline & wrist_roll_eq & ROT & \pm 30 & 2.3 \\
\hline & wrist_pitch_eq & ROT & \pm 30 & 2.3 \\
\hline
\end{tabular}

